\title{
Nutritionally Enhanced Rice to Combat Malnutrition Disorders of the Poor
}

\author{
Dr. Ingo Potrykus, Professor Emeritus
}

Major deficiency disorders, including vitamin A deficiency, are especially common in countries in which rice is the staple food. In response to the devastating effects of vitamin A deficiency, which may include blindness and, even death, "Golden Rice" has been developed to deliver this nutrient to those populations who need it most. The case of Golden Rice is used to demonstrate the challenges of radical GMO opposition, consumer acceptance, and regulation of biotechnologyderived foods.

Key words: Golden Rice, humanitarian project, vitamin A malnutrition, high-quality protein rice, high-iron rice

(c) 2003 International Life Sciences Institute

doi: 10.131/nr.2003.jun.S101-S104

\section{The Social Challenge}

Malnutrition contributes to or causes 24,000 deaths per day worldwide. Golden Rice represents a genetic engineering concept for development of nutrient-dense staple crops to help reduce malnutrition in developing countries. Major deficiency disorders concern protein/energy, iron/zinc, vitamin A, and iodine. These deficiencies are especially severe where rice is the major staple. Traditional interventions such as distribution, fortification, dietary diversification, and measures against infectious diseases are very helpful in reducing deficiency disorders, but they have not solved, and probably cannot solve, the problem. Statistics demonstrate that despite enormous efforts in applying these traditional interventions, we are still faced with, for example, 2.4 billion iron-deficient women and children and 400 million vitamin A-deficient children. Nutrient-dense staple crops offer a complement to traditional interventions in a sustainable manner. Genetic engineering has the potential to substantially enhance breeding for nutrient staple crop varieties.

Dr. Potrykus was with the Swiss Federal Institute of Technology (ETH), Zurich, Switzerland.

\section{The Scientific Response}

Protein deficiency relates both to the amount and the quality (the content in essential amino acids) of dietary protein. Rice is rich in carbohydrates (energy), but low in protein (and vitamins and minerals); rice provides, with a typical daily intake of $300 \mathrm{~g}$, only $10 \%$ of the required essential amino acids. Genetic engineering of biochemical pathways to produce an ideally balanced mixture of the amino acids isoleucine, leucine, lysine, methionine, cysteine, phenylalanine, tyrosine, threonine, tryptophan, and valine, would be beyond our technical possibilities to date. Thanks to Asp-1, a synthetic gene developed by Jesse Jaynes, and coding for an ideal high-quality storage protein (providing a balanced mixture of the amino acids), we now have the unique opportunity to approach an otherwise unfeasible task. It was, however, completely undetermined as to whether rice endosperm would provide for the necessary biochemical background to assemble such a protein and whether the genetic information would be provided and activated. To test this question, the Asp-1 gene was placed under endospermspecific control, linked to an appropriate target sequence for import into the endosperm protein storage vesicles, and transformed into rice (Japonica TP309). Surprisingly and fortunately, the Asp-1-transgenic rice plants recovered, according to western data, and obviously accumulated the Asp-1 protein in their endosperm in a range of concentrations, thus providing the essential amino acids. Detailed biochemical analysis has to verify this preliminary assumption and must show whether the concentrations achieved are nutritionally relevant.

Iron deficiency caused by a rice diet is the consequence of far too low amounts of iron in rice endosperm, the presence of an extremely potent inhibitor of iron absorption (phytate), and lack of any iron absorptionenhancing factors in a vegetarian diet. Our genetic engineering task for the endosperm was therefore to increase iron content, reduce the inhibitor, and add absorptionenhancing factors. Thus far, transgenic ferritin (from Phaseolus vulgarus) increased the iron content twofold; a transgenic metallothionin (from Oryza sativa) led to a sevenfold increase in this iron resorption-enhancing cys- 
teine-rich protein, and a transgene coding for a heatstable phytase (from Aspergillus fumigatus) produced high inhibitor-degrading phytase activity that, to date, did not maintain its heat-stability character. ${ }^{1}$ Because an integral part of our strategy is to enable phytate degradation after cooking (to leave the phytate required for germination untouched), we are now working on alternative strategies and further experiments.

Vitamin A deficiency occurs in rice-consuming populations because milled rice is totally devoid of any provitamin $\mathrm{A}$ and the people affected are too poor to afford a diversified diet. The situation is especially severe where children are raised on rice gruel. Adding provitamin A to rice endosperm required the engineering of a complete biochemical pathway, a task considered unfeasible throughout the course of our experiments. The final success was possible because of (1) the complementary expertise of two laboratories (Dr. Peter Beyer, University of Freiburg, provided the scientific knowledge and the necessary genes, and my group was specialized in genetic engineering of rice), (2) long-term public funding (e.g., Swiss governmental agencies and the Rockefeller Foundation), (3) a firm "engineering spirit" to solve the problem, and (4) "good fortune" with the biology of the rice endosperm. The introduction of transgenes for phytoene synthase (Narcissus), a phytoene/ל-carotene double-desaturase (Erwinia), and lycopene cyclase (Narcissus), to everybody's surprise, completed the biochemical pathway leading from the latest available precursor geranyl-geranyl-diphosphate to $\beta$-carotene (provitamin A). Biochemical analysis of the polished rice kernels confirmed that the "golden" endosperm color was due to varying amounts of provitamin $\mathrm{A}$ and further terpenoids of dietary interest (such as lutein and zeaxanthin). ${ }^{2}$ Assuming a high bioavailability and conversion of the $\beta$-carotene in the Golden Rice, the concentration of $1.6 \mu \mathrm{g} / \mathrm{g}$ may (according to the calculation of an experienced vitamin A nutritionist, Prof. Robert Russell, Boston) be sufficient to prevent vitamin A deficiency disorders with a daily intake of $200 \mathrm{~g}$ Golden Rice. Nutritional studies with human volunteers to test this hypothesis are in preparation. Conclusive data will, however, not be available before 2004 .

So far the novel traits "high-quality protein," "improved iron bioavailability," and "provitamin A" have been engineered into the endosperm of rice. The beauty of this genetic solution is that it can be transferred to further important crop plants such as wheat, maize, millet, sorghum, cassava, sweet potato, plantain, and legumes, etc. Because genetic engineering technology is available for all these "food security crops," it just has to be done. Unfortunately, it will not be easy to find the necessary funding and the moti- vated scientists to do it. This is especially unfortunate because no other solution would be as sustainable and as cheap!

For "normal" scientists, these successes would have been the completion of their tasks. For scientists motivated to contribute via a humanitarian project to reduction of malnutrition in developing countries, however, this is only the beginning of a series of further-and rather unusual and exhausting_challenges.

\section{The Challenge of Free Donation of Technology to Developing Countries}

The Golden Rice project was designed from the beginning as a humanitarian project for poor people in developing countries. To reach this goal and to contribute to relief from malnutrition in poor populations in developing countries, this scientific success has to be passed on to the subsistence farmers and the urban poor free of charge and limitations. To be in a position to give away the technology, we took care throughout the project to use public funding only. However, independent of our invention (which we could give away), the basic genetic engineering technology used to develop provitamin A rice used a great number of patented technologies. "Freedom-to-operate for humanitarian use," the necessary basis for variety development by partner institutions in developing countries, therefore, became a major undertaking. The inventors solved the problem thanks to an alliance with the ag-biotech industry. This is based on the agreement that transfers the rights for commercial exploitation to the ag-biotech industry, which in turn leaves the rights for noncommercial humanitarian use exclusively with the inventors, also granting the freedom-tooperate situation. The difficult problem of defining the term "humanitarian project" was solved by defining it as "income from Golden Rice per farmer or trader in developing countries below $\$ 10,000$ p.a." This definition safely includes the target population. Thanks to this agreement, the technology is now available via free licenses to public research institutions for breeding, variety development, and de novo transformation. Transfer of the technology requires signature on a sub-sub-license agreement with the inventors (not with the ag-biotech industry). Such agreements have been signed, so far, with the International Rice Research Institute (01) and PhilRice, the National Rice Research Institute (02) (Philippines); Cuu Long Delta Rice Research Institute (03) in Vietnam; Department of Biotechnology, Delhi (04), Directorate for Rice Research Hyderabad (05), Indian Agricultural Research Institute New Delhi (06), University of Delhi South Campus, New Delhi (07); Tamil Nadu Agricultural University (08) in India; Institute of Genetics, Academia Sinica, Beijing (09) and National Key Laboratory of Crop Genetic Improvement, Wuhan (10) 
in China; Agency for Agricultural Research and Development, Jakarta (11) in Indonesia; and Commonwealth Scientific and Industrial Research Institute (12) in South Africa. These institutions constitute the still-growing International Humanitarian Golden Rice Network.

\section{The Challenge of Safe Technology Transfer and Variety Development}

To ensure proper handling of the GMO material, a Humanitarian Board has been established to supervise the choice of partners, to support further improvement, to oversee needs, availability, bio-safety, and socioeconomic assessments, to coordinate the activities in the different countries, to support fundraising from public resources, to support deregulation, to facilitate exchange of information, and to mediate information of the public and generally support the humanitarian project. Members of the Board include G. Toenniessen (Rockefeller Foundation), A. Dubock (Syngenta), W. Padolina (IRRI), R.M. Russell (Tufts/USDA), H.E. Bouis (IFPRI), G. Khush (IRRI), K. Jenny (Indo-Swiss Collaboration in Biotechnology), A. Krattiger (Cornell), and the inventors P. Beyer and I. Potrykus (chairman). National and locally adapted Golden Rice varieties will be developed using either traditional breeding on the basis of selected transgenic lines, or by de novo transformation of the set of genes into popular existing varieties. Backcrossing from the experimental Japonica rice line into the essential Indica rice lines requires approximately eight generations (or three years). Direct transformation may be faster and has already been achieved into a series of Indica varieties (including the very popular Indica variety IR64) by the Vietnamese and Philippine partner institutions.

\section{The Challenge of Radical GMO Opposition and Consumer Acceptance}

Golden Rice has, unfortunately, become a key topic in the fight between proponents and opponents of plant biotechnology in food production. A radical GMO opposition is one of the last major stumbling blocks with the potential to prevent the poor in developing countries from benefiting from the project. Greenpeace and numerous other nongovernmental organizations are determined to prevent the development and use of Golden Rice. Their major reason for opposition is, obviously, the fact that they see Golden Rice as a "Trojan Horse" that opens the road for GMO technology in developing countries. In the case of Golden Rice, however, all their standard arguments used so far have been shown not to hold true. (In fact, Golden Rice benefits the poor, not industry; it has been developed in the public domain, not by industry; it will be available for the poor free of costs and limitations; subsistence farmers can use part of their harvest for the subsequent sowings; Golden Rice cultivation does not require any additional inputs; environmentalists can not conceive of realistic risks to the environment; etc.) Moreover, the public and the media have begun to understand the moral dimension of the project, so that the opposition finds itself in a difficult situation, and is therefore trying to bypass this moral dilemma by claiming that Golden Rice is useless anyhow owing to the amount that would have to be consumed by children ( $3.75 \mathrm{~kg} /$ day). This is definitely wrong, but data to prove that $200 \mathrm{~g} /$ day will be sufficient will unfortunately not be available until early in 2004 .

\section{The Challenge of Deregulation}

It is widely accepted that food derived from transgenic plants must successfully pass all requirements set up by regulatory authorities. Confronted with the realities, however, we are getting the impression that the existing regulatory framework may severely delay or even prevent the use of Golden Rice for the reduction of malnutrition-caused diseases in poor populations of developing countries. The regulatory framework has become so extensive, and the requirements so sophisticated, that the necessary financial input to carry out the required work becomes rather unrealistic for a "humanitarian" project from which no financial return is to be expected. A further severe limitation is the time factor: Golden Rice has been a reality since February 1999. Since spring 2002 the trait is available in IR64, a popular Indica rice variety grown in many developing countries. New experimental adjustments are required to render Golden Rice more amenable to the regulatory procedure. All this has the consequence that exploitation of the technology is delayed for many further years. In view of the fact that we are not discussing the sensitivities of well-fed European consumers, but the large populations whose life and health depends on contributions to sustainable reduction in malnutrition (remember: causing 24,000 deaths per day!), the question is what level of sophistication in regulation is scientifically and morally justified, and how much of it is just the consequence of weak politicians giving in to the pressure of activists who are operating, extremely successfully, on an emotional level. The Golden Rice Humanitarian Board is determined to perform the humanitarian project at the highest regulatory levels, but the question remains: is it justified to delay the use of the technology for many years for nothing else but a few minor and hypothetical risks, if the (guaranteed not hypothetical!) consequence is that many thousands are unnecessarily dying, or suffering, from severe health problems (e.g., irreversible blindness) instead of living healthy and productive lives? What is more important to our society—a strict regulatory framework for minor and 
mostly hypothetical risks, or the life and health of numerous underprivileged human beings? At least for humanitarian projects the deregulation process should relate risks to benefits! Probably, however, it is even time to reevaluate the regulatory framework to identify those features that are essential and meaningful, and delete those that are there just in response to political pressure.

We have now assembled in one plant nine transgenes to combine "provitamin A" + "high iron" + "high-quality protein" + "insect resistance." Advisors experienced in regulatory affairs inform us that it will be impossible to receive deregulation for such a transgenic plant; it will be difficult enough to get deregulation for "provitamin A" rice alone. Can we really ignore the solutions genetic engineering can provide for malnutrition in developing countries because of the sensitivities of a well-fed European minority?

1. Lucca P, Hurrell R, Potrykus I. Genetic engineering approaches to improve the bioavailability and the level of iron in rice grains. Theory Appl Genetics. 2001;102:392-397.

2. Ye X, Al-Babili S, Klöti A, et al. Engineering provitamin $A$ ( $\beta$-carotene) biosynthetic pathway into (carotenoid-free) rice endosperm. Science. 2000;287:303305. 